\title{
Nonaxisymmetric Effects in the Black Hole Accretion Inviscid Hydrodynamics: Formation and Evolution of a Tilted Torus
}

\author{
Agnieszka Janiuk ${ }^{1,2}$, Daniel Proga ${ }^{1}$ and Ryuichi Kurosawa ${ }^{1}$
}

\begin{abstract}
We report on the fourth phase of our study of slightly rotating accretion flows onto black holes. The main new element of this study is that we used fully three dimensional (3-D) numerical simulations. We consider hydrodynamics of inviscid accretion flows. We assume a spherically symmetric density distribution at the outer boundary, but brake the flow symmetry by introducing a small, latitude-dependent angular momentum. We also consider cases where angular momentum at large radii is latitude- and azimuth-dependent. For the latitude-dependent angular momentum, 3-D simulations confirm axisymmetric results: the material that has too much angular momentum to be accreted forms a thick torus near the equator. Consequently, accretion proceeds only through the polar funnel, and the mass accretion rate through the funnel is constrained by the size and shape of the torus, not by the outer conditions. In 3-D simulations, we found that the torus precesses, even for axisymmetric conditions at large radii. For the latitude and azimuth-dependent angular momentum, the non-rotating gas near the equator can also significantly affect the evolution of the rotating gas. In particular, it may prevent the formation of a proper torus (i.e. its closing, in the azimuthal direction). In such models, the mass accretion rate is only slightly less than the corresponding Bondi rate.
\end{abstract}

Subject headings: accretion, accretion discs - black hole physics - galaxies: active

\section{Introduction}

Most galactic nuclei spend a substantial fraction of their lives in an inactive ("quiescent") mode. From theoretical point of view, this inactivity is quite surprising because most galaxies, if not all, contain a super massive black hole (SMBH) at their centers (e.g., Kormendy \& Gebhardt 2001) and a large amount of gas is available for black hole accretion. Thus, one would expect vigorous accretion activity resulting in significant emission of electromagnetic radiation from all galactic nuclei, but not just from some which are referred to as active galactic nuclei (AGN).

The modeling of the inactive mode is typically based on the assumption of radiatively inefficient accretion (Ichimaru 1977; Rees et al. 1982;

\footnotetext{
${ }^{1}$ University of Nevada, Las Vegas, 4505 Maryland Pkwy, NV 89154, USA

${ }^{2}$ Copernicus Astronomical Center, Bartycka 18, 00-716, Warsaw, Poland
}

Narayan \& Yi 1994), in which the rate of accretion within the radius of influence of SMBH can be conveniently expressed by the formula derived by Bondi (1952). Some other models focus on the scenario where the accretion rate itself is much smaller than the Bondi value due to rotation, magnetic fields, or both, that can lead to convection and mass outflows (e.g., Begelman \& Meier 1982; Paczyński \& Abramowicz 1982; Narayan \& Yi 1995; Igumenshchev \& Abramowicz 1999; Blandford \& Begelman 1999; Stone, Pringle \& Begelman 1999; Quataert \& Gruzinov 2000; Machida, Matsumoto \& Mineshige 2001; Hawley \& Balbus 2002; Proga \& Begelman 2003b; Krumholz et al. 2005).

In this paper, we focus on exploring effects of gas rotation on the black hole accretion hydrodynamics, using numerical simulations of gas with simplified microphysics. We assume that gas accreting onto $\mathrm{SMBH}$ is inviscid and its specific angular momentum ranges from zero to some finite value. Such a range of specific angular mo- 
mentum is possible for instance in $\operatorname{Sgr} \mathrm{A}^{*}$ where there are many massive stars orbiting the central SMBH (Genzel et al. 2003; Schödel et al. 2003). As argued by Loeb (2004), winds from these stars might be a source of very low angular momentum gas. Our study is also relevant to other astrophysical situations, e.g., the collapsar model for long duration gamma ray bursts (GRBs) where a rotating envelope of an evolved massive star collapses onto a central compact object (Woosley 1993; Paczyński 1998; MacFadyen \& Woosley 1999; Proga et al. 2003).

Proga \& Begelman (2003a; hereafter PB03) studied the axisymmetric hydrodynamical model of the slowly rotating gas. Here we generalize this model to account for the non-axisymmetric effects. This is of a particular interest because the stellar winds at the Galactic Center are likely to feed the central black hole in a non-axisymmetric way (e.g., King et al. 2005; Volonteri et al. 2007). However, non-axisymmetric effects can be important even for the axisymmetric initial and outer boundary conditions because of hydrodynamical instabilities. PB03 showed that a pressure/rotation supported torus forms around the black hole and the accretion rate is smaller than the Bondi rate. In the inviscid case, accretion is possible only through the polar funnels where gas does not rotate or rotates very slowly. Our goal is to check whether this result holds in 3-D and to what extend when for example, a non-uniform initial distribution of the specific angular momentum is assumed. when there is a gas with zero angular momentum at the equator. One can expect that if the amount of the non-rotating material near the equator is large enough, the torus may not form and the accretion rate would not be much smaller than the Bondi value. One of our main questions is whether the accretion can proceed through the equatorial plane or it can proceed only through the polar funnels as in an axisymmetric case.

The content of the article is the following. In Section 2, we describe the method used in our calculations. In Section 3, we present the simulations results. First, we discuss the test runs performed with the new version of ZEUS-MP code (Sec. 3.1), then, we describe the results for the initially axisymmetric $3-\mathrm{D}$ case, which is our 'reference' model (Sec. 3.2), and finally we present the results for models with non-axisymmetric ini- tial conditions (Sec. 3.3). We discuss our results in Section 4.

\section{Method}

In our calculations we use the 3 -D code ZEUSMP (Stone \& Norman 1992; Hayes \& Norman 2003). The code solves the equations of hydrodynamics:

$$
\begin{gathered}
\frac{d \rho}{d t}+\rho \nabla \mathbf{v}=0 \\
\rho \frac{d \mathbf{v}}{d t}=-\nabla P+\rho \nabla \Phi \\
\rho \frac{d}{d t}\left(\frac{e}{\rho}\right)+P \nabla \mathbf{v}=0
\end{gathered}
$$

where $\rho$ is the gas density, $e$ is the internal energy density, $P=(\gamma-1) e$ is the gas pressure, and $\mathbf{v}$ is the velocity of the flow. We adopt the ratio of specific heats to be $\gamma=5 / 3$. We modified the ZEUS-MP code to use the pseudo-Newtonian gravitational potential (Paczyński \& Wiita 1980):

$$
\Phi(r)=-\frac{G M}{r-R_{\mathrm{S}}}
$$

where $R_{S}=2 G M / c^{2}$ is the Schwarzschild radius. We start the simulation with the spherical Bondi accretion solution (PB03), derived iteratively for the density, $\rho(r)$, energy, $e(r)$, and radial velocity $v_{\mathrm{r}}(r)$ distributions. We express the accretion rate resulting from simulation in the units of the Bondi accretion rate:

$$
\dot{M}_{\mathrm{B}}=\lambda 4 \pi \frac{G^{2} M^{2}}{c_{\infty}^{3}} \rho_{\infty}
$$

where $\lambda \approx 0.29$ (see e.g. PB03 for the exact expression for $\lambda$ ).

The initial velocity $v_{\theta}$ is set zero everywhere, whereas the velocity $v_{\phi}$ is initially non-zero only in a fixed, quasi-conical zone, with a limited radial size. This zone of initial rotation is formally defined as follows:

$v_{\phi}=\left\{\begin{array}{lll}0 & \text { for } & -v_{\mathrm{r}}>c_{\infty} \\ \sqrt{l_{0}} R_{B} c_{\infty} \frac{1-|\cos \theta|}{r \sin \theta} & \text { for } & -v_{\mathrm{r}}<c_{\infty}\end{array}\right.$ and $180^{\circ}-\frac{\Delta \phi_{0}}{2}<\phi$

where $l_{0}=0.1$ is a dimensionless model parameter. The other model parameters are: $c_{\infty}, \rho_{\infty}$, $M_{\mathrm{BH}}, r_{\mathrm{in}}$, and $r_{\mathrm{out}}$. The Bondi radius is equal 
to $R_{\mathrm{B}}=G M / c_{\infty}^{2}$, and in our simulation it is equal to $1000 R_{\mathrm{S}}$. Our computational zone extends from $1.5 \times 10^{-3} R_{\mathrm{B}}$ to $1.2 R_{\mathrm{B}}$. When presenting the results, we use the units of $R_{\mathrm{B}}, c_{\infty}, \rho_{\infty}$ and time $t^{\prime}=t / t_{\text {orb }}\left(R_{\mathrm{B}}\right)$. We perform our simulations for several values of the parameter $\Delta \phi_{0}$. We use the spherical coordinate system, RTP, and the boundary conditions in the $r, \theta$ and $\phi$ directions are outflow, reflection and periodic, respectively. The resolution in $r$-direction was 140 zones, with $d r_{\mathrm{i}+1} / d r_{\mathrm{i}}=1.05$, in $\theta$-direction it was 96 or 100 zones, and in $\phi$-direction we had 10, 32 or 60 zones, with $d \theta_{\mathrm{j}+1} / d \theta_{\mathrm{j}}=d \phi_{\mathrm{k}+1} / d \phi_{\mathrm{k}}=1.0$. We note here that because of a moderate resolution in the $\phi$ direction, the simulations are able to capture only the lowest few non-axisymmetric modes.

\section{Results}

\subsection{Test runs}

Initially, we performed test runs, to check whether the spherical or axial symmetry is conserved wherever it should be conserved, and whether the 2-D results of the axisymmetric calculations are reproduced in 3-D. Having calculated both the models of spherical Bondi accretion (smodels), as well as the axisymmetric accretion with low angular momentum (l-models), we found that:

(a) the results from the 2-D models are reproduced in the $r-\theta$ slices of the 3-D models; in particular, the accretion rate $\dot{M}_{\text {in }}$ and the flow pattern are the same;

(b) the non-radial velocity components in the smodels, which analytically should be $v_{\theta}=v_{\phi}=0$, can locally have non zero values, but both are orders of magnitude smaller than the local radial velocity and sound speed (that ratio is of the order of $10^{-10}-10^{-13}$ );

(c) the symmetry with respect to both the equatorial plane and rotation axis is conserved $(\delta x / x \leq$ $10^{-7}$, where $x$ denotes density, energy, or velocity components) in all models at early stages of the evolution, i.e. $t^{\prime}<0.033$.

The s-models conserved their spherical symmetry in both 2-D and 3-D, throughout our runs. For l-models, as the system evolved, asymmetries began to arise with respect to the equatorial plane, in both 2-D and 3-D models. We note that this was already the case in PB03 simulations. The axisymmetry in the present 'reference' 3-D model (i.e. the model with axisymmetric initial conditions) is conserved initially and at intermediate times of the system evolution (however see the results below).

\subsection{Time evolution of the initially ax- isymmetric flow}

The computations of an axisymmetric, 2.5-D model of an accretion flow with low angular momentum were presented in PB03. We have recalculated this model in 3-D for the purpose of the present work. The simulations start from a spherically symmetric gas cloud around a black hole, with density and velocity distributions derived from the Bondi solution. The matter located far from the black hole possesses a specific angular momentum that exceeds the critical value, $l_{\text {crit }}=2 R_{\mathrm{S}} c$, at the equatorial plane and is decreasing towards the polar regions (cf. Eq. 6).

The time evolution of the system with the initial conditions described above proceeds as follows. After a transient episode of purely radial infall, when the rotating material reaches the vicinity of the black hole, a thick torus forms in the equatorial region. The gas settled in this region is supported against gravity by the gas pressure and rotation, and the rate of accretion on the black hole, $\dot{M}_{\text {in }}$, decreased down to about $30 \%$ of the Bondi accretion rate (in PB03 the exact value of $\dot{M}_{\text {in }}$ was found to depend on the details of the angular momentum distribution). This is because the material accretes only through the polar funnels, while the torus is made of material that cannot accrete. (There is no transport due to viscosity; however the non-axisymmetric shocks can result in some transport of the angular momentum). The gas which approached the centrifugal barrier at the equator, could either outflow radially, or try to turn towards one of the poles and accrete. Consequently, meridional circulation movements are observed in the flow.

The accretion rate onto $\mathrm{BH}$ varies in time. Due to meridional circulations, the flow is not symmetric with respect to the equatorial plane; however, the time averaged properties (e.g. accretion rate) and the shape of the torus did settle down to a steady state, as shown in PB03. In the equator the flow is subsonic down to very small radii, while at the poles, at some distance from the center, the 
radial velocity exceeds the local speed of sound.

Here, we repeated the calculations of PB03 in $3-\mathrm{D}$ and ran new simulations on a relatively long time scale. Most of our simulation runs lasted up to $t^{\prime}=0.36$. For comparison, the sound crossing time at the $R_{\mathrm{B}}$ is about $t_{\text {sound }}^{\prime}\left(R_{\mathrm{B}}\right)=0.125$, while at the radii corresponding to the dense tori formed in the innermost part of the flow (see Section 3.3), the sound crossing time is $t_{\text {sound }}^{\prime}\left(R_{\text {torus }}\right) \sim 10^{-3}$.

Figure 1 shows the sonic surface, i.e. the isosurface where the Mach number, $M_{\text {tot }}=$ $\sqrt{\left(v_{r}^{2}+v_{\theta}^{2}+v_{\phi}^{2}\right) / c_{s}^{2}}$ is a unity. at the $t^{\prime}=0.1$. For the zero-vorticity flow, the surface shape can be derived analytically, as it passes orthogonally through the velocity equipotential surfaces (Papaloizou \& Szuszkiewicz 1994). In general, the shape of the surface can be more complex. As one can see in the Figure, the sound waves are propagating outwards in the flow. The sonic surface for initially axisymmetric model is shown in the left panel, and for comparison in the right panel we also show the non-axisymmetric case (model $A$, to be described in Sec. 3.3.

We find that both qualitatively and quantitatively the initial results in 3-D axisymmetric case are the same as in 2.5-D. The main new feature of the torus in 3-D, which could not be studied in the PB03 simulation, is that in the very late stages of the evolution $\left(t^{\prime}>\sim 0.3\right)$ the axisymmetry breaks and the inner torus becomes tilted with respect to the equator, and in the end it starts precessing. This departure from the symmetry at later times is caused by the equatorial outflow and meridional circulations in the torus become suppressed, while the material tries to get through towards the black hole and accrete along one of the poles. At the same time, for some regions (e.g., $\left.\phi \sim 0^{\circ}\right)$ the accretion occurs through the northern pole, at the opposite side $\left(\phi \sim 180^{\circ}\right)$ and the flow chooses rather the southern pole. Consequently, a torque is induced and the rotation axis of the torus changes in time.

The precession of the torus is illustrated in Figure 2 in terms of the total angular momentum, $L_{\text {tot }}$, which is changing in time. This figure shows the motion of the innermost part of the flow, i.e. the torus, defined by the density threshold $\rho_{\text {min }}=500$. Initially, the dominant component is $L_{\mathrm{z}}$, while $L_{\mathrm{x}}$ and $L_{\mathrm{y}}$ are close to zero (but fluc- tuating). It means that the torus rotates basically around the $z$ axis. After $t^{\prime} \sim 0.21, L_{\mathrm{x}}$ and $L_{\mathrm{y}}$ are non-zero, and the rotation axis tilts towards the 4 th quarter in the $x-y$ plane. At $t^{\prime} \sim 0.3, L_{\mathrm{y}}$ starts decreasing while $L_{\mathrm{x}}$ still decreases and $L_{\mathrm{z}}$ is almost constant. It means that the torus is now precessing, i.e. the rotation axis moves counterclockwise with respect to an observer along the $+z$ axis. Figure 2 also shows for comparison, the results for the torus precession in case of nonaxisymmetric initial conditions (model $A$ ). This model will be discussed below in more detail (Sec. 3.3 .

The precession is also shown in Figures 14 and 15 (see e.g. Fragile et al. 2007). The tilt, defined as an angle between the angular momentum vector of the gas and the $z$ axis, is initially equal to zero for all radii. Later during the simulation the tilt rises strongly in the inner parts of the flow. The tilt is equal to:

$$
\beta(r, t)=\arccos \left(\frac{L_{z}}{L}\right)
$$

In the Figure 14 we plot the tilt angle, $\beta(r, t)$, as a function of radius, for several time snapshots, starting from $t^{\prime}=0.18$. The differential form of the torus precession is also visible in Figure 15 , in which we plot the cumulative twist angle, $\gamma(r, t)$, as a function of time. The twist angle is defined as a cumulative angle by which the angular momentum vector revolves in the $x-y$ plane, by the time $t$ :

$$
\gamma(r, t)=\arccos \left(\frac{L_{x}}{\sqrt{L_{x}^{2}+L_{y}^{2}}}\right)
$$

Before the disk was tilted, it did not precess, and by definition the twist was zero. Therefore the results in the Figure are also plotted from time $t^{\prime}=0.18$. The solid line is the twist averaged over the radius for the inner part of the flow $\left(1.5 \times 10^{-3} R_{\mathrm{B}}<r<5 \times 10^{-2} R_{\mathrm{B}}\right)$, i.e. the torus, while the dashed line shows the twist averaged for the whole range of radii. Clearly, the innermost torus precesses much stronger than the rest of the flow, and the maximum twist at the end of the simulation was $\gamma \approx 120^{\circ}$.

To check whether the tilt and precession of the inner torus found in this simulation is a physical or rather numerical effect, we performed several 
further test simulations. First, we reversed the direction of the flow in the initial condition, i.e. we changed the sign of the azimuthal velocity $v_{\phi}$. In this simulation, we also found that the torus tilts and starts precessing, at the same time $\left(t^{\prime}>\right.$ $0.18)$. However, the tilt and precession are in the opposite directions as measured with respect to the grid, i.e. the initial tilt was $\beta=180^{\circ}$ and decreased to about $\beta=150^{\circ}$, while the twist was negative.

Second, we checked how transient the effect of precession is. Due to technical limitations, we were not able to run all the simulations for very long time, but we completed one run up to $t^{\prime}=0.8$, for the model $R_{32}$. In this model we found that the tilt angle increased from $\sim 30^{\circ}$ to $\sim 40^{\circ}$, and the tilt spreaded to larger radii, so that not only the innermost parts of the flow precessed. However, at this very late phase, some of the ring-like structures of the largest density were broken into two separate parts, each of them of a "C" shape. Therefore the precession may not be a long-term effect. We plan to investigate this in near future.

Third, we checked for the importance of the adopted physics of the model, in particular, the ratio of the sound speed to the free fall velocity. We calculated two models with much smaller Bondi radius, $R_{\mathrm{B}}=100 R_{\mathrm{S}}$ and $R_{\mathrm{B}}=300 R_{\mathrm{S}}$, which correspond to a much larger sound speed at infinity: respectively, 3.15 and 1.82 times larger than in all the other simulations. These models are denoted as $M_{100}$ and $M_{300}$ in Table 1 . The models could be run for much longer time in terms of $t_{\mathrm{orb}}\left(R_{\mathrm{B}}\right)$, i.e. for $t^{\prime}=11$ and $t^{\prime}=2$, respectively. However, in these models we did not find any signatures of tilt or precession. This is because here the Mach numbers are never large: they are at most $M=3.3-3.5$ at the inner radius, whereas for the precessing torus the Mach numbers reached the values as large as 4.7 - 6.0. The small Mach numbers in models $M_{100}$ and $M_{300}$ make the shocks smaller; hence they do not amplify the asymmetries growing out of initial perturbations.

Finally, we tested the role of the artificial viscosity, which might help to spread the shocks and avoid precession if it was a numerical artifact. The artificial viscosity was parametrized with the standard Neumann-Richtmeyer artificial viscosity coefficient $q$ con $=2.0$ However, the results in this simulation were very similar to the original simulation. Specifically, for time $t^{\prime}=0.324$ the maximum tilt angle was $26^{\circ}$, while in the former case and $28^{\circ}$ in the latter.

From the above tests, we conclude that the precession in our initially axisymmetric model is rather a physical than numerical effect, and is connected with relatively large supersonic speeds of the flow achieved in our model.

\subsection{Time evolution for the non-axisymmetric initial conditions}

Now we investigate how the non-axisymmetric initial distribution of the specific angular momentum affects the evolution of the flow. In particular, we check whether the rotationally supported torus forms and if a steady state can be achieved (with or without torus precession). We start our simulation with the non-zero specific angular momentum enclosed in a conical region of a width $\Delta \phi_{0}$ (see Eq. 6). A naive prediction could be that the rotating gas will reach the innermost regions, spiral in, and after a few orbital cycles the material with large angular momentum would be mixed with the non-rotating gas. Therefore a rotationally supported torus would form, regardless of $\Delta \phi_{0}$. The only dependence on this parameter would be the moment when such a torus forms. However, as we show below, the numerical simulations lead us to a different result: depending on $\Delta \phi_{0}$ the rotating gas may not form a torus at all.

We performed the runs for non-axisymmetric initial conditions for a range of $\Delta \phi_{0}$. The models are summarized in Table 1 . The non-axisymmetric models are labeled with the letters $A-E$, while the reference model is labeled as $R$. As we mentioned in Sec. 2 and as the Table shows, we tested the models with smaller (32 zones) and larger (60 zones) resolution in the $\phi$-direction. We checked, that the time averaged results for the accretion rate only very weakly depend on the resolution, however the amplitude of time variability of $\dot{M}$ increases with resolution.

Below, we present these results for the largest adopted value of $\Delta \phi_{0}=330^{\circ}(\operatorname{model} A)$. This parameter translates into a small non-axisymmetric perturbation in the initial conditions, i.e. small content of non-rotating material.

In Figures 3, 4, 5, 6, and 7 we show the color 
coded maps of the central region, as well as the zoomed-out. The maps show the density distribution and velocity field, as well as the specific angular momentum, plotted for several snapshots during the evolution: $t^{\prime}=0,0.018,0.09,0.16,0.23$ and 0.29. Note that the top-right panel in Figure 3. as well as all the top panels in Figure 6, i.e. for $l_{\text {spec }}(t=0)$, are plotted on the scale 20 times larges compared to other panels, because in the inner region initially we assumed $l_{\text {spec }}=0$.

Figure 3 shows the density and specific angular momentum distribution in the central region (up to $0.02 R_{\mathrm{B}}$ ), plotted for the equatorial plane. The orientation of the plots in the $x-y$ plane is standard, i.e. the $x>0$ semi-axis corresponds to $\phi=0$. The density distribution, initially spherical at $t=0$ (top left panel) changes in time, as the material which carries specific angular momentum approaches the center. At $t^{\prime}=0.018$, the gas is rotating around the $z$-axis (arrows over plotted on the density maps denote the direction of the velocity vectors with components $v_{\mathrm{r}}$ and $v_{\phi}$ ). The material is distributed axisymmetrically, and the specific angular momentum is rather large (i.e. $\left.0.8<l_{\text {spec }} / l_{\text {crit }}<1.2\right)$ for most of the $\phi$ directions. A clump of gas with relatively smaller $l_{\text {spec }}$, which can be seen on the second right panel, is a remaining of the gas with $l_{\text {spec }}=0$ initially present at the equator for $\phi=\left(-15^{\circ}, 15^{\circ}\right)$. This clump is tracking the archimedean spiral (see also e.g. Lemaster et al. 2007), and such a trajectory appears to be due to the pressure gradient force and rotation (similarly to a cyclone).

At $t^{\prime}=0.09$, the material with small $l_{\text {spec }}$ is already mixed with the gas of high $l_{\text {spec }}$. The material rotates very fast in the equatorial plane, and a circular pattern of slightly larger and smaller specific angular momentum regions, visible on the third plot, form due to mixing. These motions are suppressed at $t^{\prime}=0.16$. For some specific directions, namely $\phi \sim 90^{\circ}$ and $\phi \sim 270^{\circ}$, the angular momentum near the inner radius becomes very small (as indicated by the green spots in Fig. 3), and the density in these regions drops, indicating that the material falls radially to the black hole.

The solid line in the angular momentum maps mark the contour at which $l_{\text {spec }} / l_{\text {crit }}=1.0$. We note that at $t^{\prime}=0.23$ and $t^{\prime}=0.29$, in the equatorial plane a substantial fraction of material has $l_{\text {spec }}<l_{\text {crit }}$. This material is located at an ex- tended region approximately along the diagonal of the plane (i.e. $\phi \sim 45^{\circ}$ and $\phi \sim 225^{\circ}$, as marked by the orange shade in Fig. 3). Another region of even smaller angular momentum, $l_{\text {spec }} \ll l_{\text {crit }}$, has a very limited radial extension close to the center, and is slightly elongated along the other diagonal (i.e. $\phi \sim 135^{\circ}$ and $\phi \sim 315^{\circ}$, as marked by the green shade in Fig. 33. For the same $\phi$, but at larger distances, the specific angular momentum in the equatorial plane is very large, and exceeds $l_{\text {crit }}$.

In Figure 4 we show the distribution of $l_{\mathrm{spec}}$ on the larger scale. (Note also that the color scale is different than in Fig. 3. The red and green shades now mark the regions with $l_{\text {spec }} / l_{\text {crit }}>1.0$ ). As the Figure shows, more material with $l_{\text {spec }}<l_{\text {crit }}$ mixes in at late stages of evolution, $t^{\prime}=0.09 \mathrm{sec}$.

From the Figs. 3 and 4 , we conclude that a torus starts forming in the innermost regions in the equatorial plane at $t^{\prime}=0.018$, and it is rotationally supported for all the $\phi$ directions, because the low $l_{\text {spec }}$ material is quickly mixed in. At $t^{\prime}=0.09$, the distribution of density and specific angular momentum is almost axisymmetric, but only in the inner region. However as the zoomout maps show, the gas with $l_{\text {spec }}<l_{\text {crit }}$ is mixing in and is distributed asymmetrically. The innermost symmetric configuration lasts until about $t^{\prime}=0.16$, and after this time the torus position and shape changes. To investigate what really happens, we need to look at the flow from a different perspective. Therefore, in Figures 5, 6 and 7) we show the slices perpendicular to the equatorial plane. The maps show density and velocity field, as well as the specific angular momentum, as seen from $\phi=0^{\circ}, 90^{\circ}, 180^{\circ}$ and $270^{\circ}$, at the same times as in Figs 3 and 4

As shown in the Figure 5, at $t^{\prime}=0.018$, the material is indeed accumulated near the equator, while at the poles the density is much lower. The axial symmetry is not perfect, since at $\phi=180^{\circ}$ the torus is geometrically thicker than at other $\phi$ directions, which corresponds to the location of the 'clump' with smaller specific angular momentum (c.f., Fig. 3). As indicated by the arrows (velocity vectors with $v_{\mathrm{r}}$ and $v_{\theta}$ components), an equatorial outflow occurs at most of the $\phi$ directions, however at $\phi=180^{\circ}$ this outflow is weaker, because of slower rotation. 
At $t^{\prime}=0.09$, the torus is relatively thin and located at the equator in every $\phi$ direction, while at the poles the density is very low. The gas accretes onto the center mostly through the poles, while at the equator the flow pattern is complex (circulations, outflows). The flow complexity is reduced at $t^{\prime}=0.16$, because the gas that flows into the equatorial region has less angular momentum and can directly accrete onto the black hole, finding its way along one of the poles. At $\phi=0^{\circ}$ and $\phi=270^{\circ}$ the gas turns towards the northern pole (i.e. $+z$ ), while at $\phi=180^{\circ}$ and $\phi=90^{\circ}$ the flow turns towards the southern pole (i.e. $-z$ ). A line which would mark the regions of the maximum density, is now tilted with respect to the equator by an angle of $\sim 20^{\circ}$. This means that the torus which at $t^{\prime}=0.09$, was almost symmetric with respect to the equatorial plane, is tilted after $t^{\prime}=0.16$.

In Figure 6 we show the maps of specific angular momentum, also perpendicularly to the equatorial plane and for the same $\phi$ directions as in Fig. 5. As the Figure shows, the specific angular momentum distribution is symmetric with respect to the equatorial plane at $t^{\prime}=0.09$, and nearly symmetric at $t^{\prime}=0.16$. The axial symmetry is not perfect, however the regions of large angular momentum $\left(l_{\text {spec }} \geq l_{\text {crit }}\right)$ appear in every slice. At $t^{\prime} \geq 0.16$, the flow is no longer symmetric, and the regions with very large specific angular momentum appear either below the equator (at $\phi=0^{\circ}$ and $\left.\phi=90^{\circ}\right)$ or above it $\left(\right.$ at $\phi=180^{\circ}$ and $\phi=270^{\circ}$ ). These regions correspond to a flatter torus, i.e. relatively thin on one side, while the gas which is not rotating fast makes the configuration geometrically thicker. We notice, that the lack of the top-bottom symmetry in density and $l_{\text {spec }}$ maps at this phase of system evolution is a consequence of the earlier non-axisymmetry. This asymmetry was introduced to the velocity field in the initial conditions at large radii, and subsequently propagated to the inner radii and affected the density distribution there, as soon as the rotating gas reached there.

In Figure 7 we show the distribution of the specific angular momentum in the zoom out. The flow is rotationally supported in the outer regions. The $l_{\text {spec }}$ distribution in the flow is asymmetric at large scales (there is significantly more material with large $l_{\text {spec }}$ for the directions of $\phi=0^{\circ}$ and $270^{\circ}$ than for $\phi=90^{\circ}$ and $\left.180^{\circ}\right)$. However, this is the case only in the inner region, i.e. the torus is tilted, while outer regions, even at late times, are rather symmetric with respect to the equatorial plane.

To visualize the 3 -D configuration better, in Figure 8 we show the density isosurfaces in 3-D. The plots show 3 arbitrarily chosen contours of the constant density: 2000, 1250 and $500 \rho_{\infty}$, which correspond to the gas densities very close to the inner radius, i.e. inside $\sim 0.004 R_{\mathrm{B}}$. The maps are plotted for 5 different time snapshots: $t^{\prime}=0.018$, $0.09,0.16,0.23$ and 0.29 . We do not show the density distribution at $\mathrm{t}=0$, because it is purely spherical. The orientation of the figures is almost edge-on, i.e. the $z$-axis is the rotation axis of the system, and $x-y$ plane is the equatorial plane.

These density contours may be regarded as the shapes of the torus (however one should keep in mind that the material with smaller/larger density is also present there). Therefore, as the Figure shows, the torus is closed already at $t^{\prime}=0.018$. After $t^{\prime}=0.16$, the configuration becomes tilted with respect to the equatorial plane, and at $t^{\prime}=$ 0.29 this tilt is the largest. After $t^{\prime}=0.29$ the torus starts precessing, and the precession period is very long (by the end of the run, the ring precessed around the $z$-axis by less than $90^{\circ}$ ). The torus precession was also shown in the right panel of the Figure 2 (Sec. 3.2). The changing values of $L_{\mathrm{x}}, L_{\mathrm{y}}$ and $L_{\mathrm{z}}$, show that at $t^{\prime} \sim 0.1$ the rotation axis, which is defined by the direction of $\mathbf{L}$, starts to tilt towards the first quarter of the $x-y$ plane, and after $t^{\prime} \sim 0.29$ the rotation axis moves counter-clockwise.

In Figure 9 we show the isosurfaces of the specific angular momentum in 3-D. The contours are for $l / l_{\text {crit }}=1.3,2.15$ and 3.0, and correspond to the zoomed-out regions shown in Fig. 7 (the radial extension of about $0.6 R_{\mathrm{B}}$ ). As the Figure shows, the material with various angular momentum is mixing in the innermost region at $t^{\prime}=0.018$ while at the outer parts initially the momentum is not mixed. At later times, the outer parts of the flow contain more and more mixed angular momentum layers, while in the innermost region the gas rotates slower than at the beginning, and the angular momentum distribution is smoother.

In Figure 10 we show the maps of entropy, $S$, radial to azimuthal velocity ratio, $v_{\mathrm{r}} / v_{\phi}$, angular velocity, $\Omega$, and velocity divergence, $\operatorname{div} \mathbf{v}$, as cal- 
culated close to the end of this simulation, at time $t^{\prime}=0.29$. The maps show the inner region in the equatorial plane. Close to the center, the angular velocity is the largest along the diagonal of the plane $\left(\phi \sim 45^{\circ}\right.$ and $\left.\phi \sim 225^{\circ}\right)$, which corresponds to the cross-section line along which the torus crosses through the equatorial plane (cf. Fig. 8). This line also corresponds to the largest entropy, as well as positive velocity divergence, while at the other diagonal there is smaller entropy and negative velocity divergence. This means that the fluid is compressible (and supersonic).

Our analysis of the $3-\mathrm{D}$ results shows the flow has a largest negative divergence close to the poles while the largest positive divergence is somewhat above and below the equator, i.e. on the surface of the torus, as well as at its cusp. From the poles, the gas flows radially onto the center with large supersonic velocities, i.e. $M_{\mathrm{r}}=\sqrt{v_{\mathrm{r}}^{2} / c_{\mathrm{s}}^{2}} \gg 1$. Close to the equator, the flow is captured in the torus and the radial velocities are smaller, so $M_{\mathrm{r}}<1$. Still, the gas rotates very fast, and the total Mach number is large, $M_{\text {tot }} \gg 1$, because of the contribution from the azimuthal velocity.

The entropy in the flow should be constant along the streamlines, however may vary from one streamline to the other. The entropy gradient corresponds to a non-zero vorticity in the flow. The direction of vorticity arrows $(\mathbf{w}=\operatorname{rot} \times \mathbf{v})$ in the bottom-right panel indicate that the torus is rotating counter-clockwise. The direction of radial velocity arrows in the bottom-left panel confirms that the radial inflow occurs from the directions of the smallest angular velocity.

The above considerations led us to the conclusion that qualitatively, the basic pattern of the torus evolution is uniform. It does only weakly depend on whether we assume the axisymmetric initial conditions, or if the initial distribution of angular momentum was perturbed (provided that this perturbation was small enough to allow for the torus formation). The axisymmetry was explicitly assumed by PB03 throughout their 2-D simulations, as well as in our 3-D reference models in the initial conditions. The similarity of the torus behavior that we find here means that in both $3-\mathrm{D}$ models $R$ and in the $A$ models, the rotationally supported tori form after time $t^{\prime}=0.018$, than they exhibit strong equatorial outflows (which stabilize them), then they become tilted with respect to the equatorial plane due to the asymmetric polar accretion, and finally start precessing. What differs in the models, is the moment when the outflow stops and when the torus becomes tilted, as well as the tilt angle (it is about twice as large in model $A$ than in model $R$ at the end of our simulations; see also Fig. 22. These two features (i.e. tilt and precession) cannot be studied in the 2-D simulations, but are detected in the 3-D models.

However, we note that the similarity between the $R$ and $A$ models is less pronounced when considering the details, e.g., of the torus shape. The asymmetric torus can be thicker (warped), or thinner, depending on $\phi$. To investigate further the difference between $R$ and $A$ models and the role of non-axisymmetry, we calculated the angular momentum at the inner boundary, as a function of the angles $\theta$ and $\phi$.

In the axisymmetric model $R$, the specific angular momentum at $r_{\text {in }}$ depends only on $\theta$ by definition. After the torus is formed, the maximum value at the equator is $l_{\text {spec }} / l_{\text {crit }}\left(r=r_{\text {in }}, \theta=\right.$ $\left.90^{\circ}\right) \approx 0.85$, while the value averaged over the angle $\theta$ is $\bar{l}\left(r=r_{\text {in }}\right) \approx 0.55$. For the nonaxisymmetric case (in particular, model $A$ ), the results depend also on the angle $\phi$. Therefore both the equatorial angular momentum, $l\left(r=r_{\mathrm{in}}, \theta=\right.$ $\left.90^{\circ}\right)$, and the $\theta$-averaged, $\bar{l}\left(r=r_{\text {in }}\right)$, are scattered and do not have to match with the axisymmetric solution. In Figure 11, the results for the axisymmetric model, are denoted by single points, while the non-axisymmetric solutions are represented by the horizontal lines to show the scatter in $\phi$.

The level of non-axisymmetry is represented as the spread between the maximum and minimum values of $l_{\text {spec }}$ at a given time. For the model $A$, in the beginning of the torus evolution, i.e $0.018<t^{\prime}<0.09$, the equatorial and averaged values of $l_{\text {spec }}$ match well with the axisymmetric solutions and are only slightly scattered with $\phi$. The equatorial value is much larger than the $\theta$ averaged, which means that the torus is located in the equatorial plane where $l_{\text {spec }}$ is the largest. As the evolution proceeds, $t^{\prime} \geq 0.09$, the scatter with $\phi$ increases and the range of $l_{\text {spec }}$ does not match the axially symmetric solution. However, the equatorial angular momentum is still much larger than the average. This means that the torus is located at the equatorial plane, but it is now asymmetric. After $t^{\prime} \geq 0.16$, the situa- 
tion changes, and $l_{\text {spec }}$ at the equator has a very large scatter, being either smaller or larger than the $\theta$-averaged value (i.e. the solid and dashed lines overlap in the Figure). The averaged angular momentum has also some scatter, but much smaller than the equatorial one. This indicates that the torus is not symmetric, and is tilted with respect to the equatorial plane.

We performed similar analysis of other nonaxisymmetric models, $B-E$ (cf. Table 1). For models $B$ and $C\left(\Delta \phi_{0}=240^{\circ}\right.$ and $\left.\Delta \phi_{0}=120^{\circ}\right)$, at the beginning of the simulation $0.018<t^{\prime}<$ 0.07 , the equatorial value of $l_{\text {spec }}$ is always larger than the averaged, and the scatter with $\phi$ is smaller in model $B$ than that in model $C$. This indicates that a rotationally supported torus is present in the equatorial plane. At later times, the equatorial $l_{\text {spec }}$ becomes equal or smaller than average, and the scatter in both models $B$ and $C$ is quite substantial (larger than in model $A$ ). This implies that a torus, which possibly tried to form at the early phase, is broken (i.e. not completely closed), as well as tilted from the equator. An example of such a 'broken torus' configuration is shown in Figure 16.

For models $D$ and $E\left(\Delta \phi_{0}=30^{\circ}\right.$ and $\Delta \phi_{0}=$ $\left.60^{\circ}\right)$, the scatter in both $\theta$-averaged and equatorial $l_{\text {spec }}$ is very large and does not decrease with time (up to $t^{\prime} \sim 0.018$ ). The averaged $l_{\text {spec }}$ can be larger or smaller than the axisymmetric one, while the equatorial $l_{\text {spec }}$ in these models is always smaller than that in the axisymmetric case, and locally (i.e. for some $\phi$ angles) can be smaller than the $\theta$-averaged. We conclude that in these models the torus does not form, the solution is not axisymmetric, and the gas with very small angular momentum can accrete onto the black hole through the equator.

We note that in this sense the properties of these models are similar to those of the model $A$ in later times. However, when comparing the density distributions, in the models $D$ and $E$ the gas is always distributed much more uniformly, i.e. it does not concentrate neither close to the equator nor to any specific plane. In model $A$, the gas density near the poles is always orders of magnitude lower than that at the equatorial plane, or the plane tilted to the equator by a small angle.

This is not the case for models $D$ and $E$, for which the torus does not form. For models $B$ and $C$, the gas is concentrating near the equator, but only for some range of $\phi$-directions, i.e. the torus is not closed.

Figure 12 shows the time evolution of the accretion rate through the inner boundary, $\dot{M}_{\text {in }}$ for the non-axisymmetric models. Before the rotating material approaches the black hole $\left(t^{\prime}<0.018\right)$, $\dot{M}_{\text {in }}$ is equal to the Bondi accretion rate for all models. Once the gas starts rotating also in the innermost parts, the accretion rate drops reaching about $25 \%-40 \%$ of the Bondi rate for models $A$, $B, C$ and $R$. This is because much more material gets captured in the rotating torus, and does not fall radially into the black hole. The model $A$ gives the lowest accretion rate with rather small and regular variability pattern, very close to that obtained in the reference model $R$.

Figure 13 presents the evolution of the angular momentum flux through the inner boundary: $\dot{L}_{\text {in }}=\int l_{\text {spec }} \rho v_{r} d s$, in units of the critical angular momentum $l_{\text {crit }}$ and renormalized by the value of the Bondi accretion rate. At the beginning of the simulation, $\dot{L}_{\text {in }}=0$, since there is no rotation in the vicinity of the black hole. Once the rotating matter reaches the inner boundary, the angular momentum starts accreting to the center. When the torus starts forming, the fast rotation near the center leads to a fast rise in $\dot{L}_{\text {in }}$. However, after several orbital cycles the outflow begins and the net radial velocity drops, as well as drops the density near the polar regions, therefore the $\dot{L}_{\text {in }}$ is rather small during the torus evolution.

Note that the quantity plotted in the Figure 13 is not a flux of specific angular momentum, but the total one. This corresponds to the amount of angular momentum which may be transferred to the black hole and used to spin it up. However, as the Figure shows, the total angular momentum which the black hole could gain during our simulation, is extremely low: $a=(c J) / G M^{2} \approx 4 \times 10^{-6}-10^{-5}$, where $J=\dot{L}_{\text {in }} \Delta t$.

Figure 13 shows two trends in the magnitude of $\dot{L}_{\text {in }}$. For small $\Delta \phi_{0}$ (models $E$ and $D$ ), the rotation at inner boundary is very small, but the density is high both in the equator and in the polar regions (still close to the spherical accretion). Therefore, in model $D$, with faster rotation, $\dot{L}_{\text {in }}$ is larger. For large $\Delta \phi_{0}$ (models $A, B$ and $C$ ), the material accumulates rather close to the equator, 
at least for some $\phi$ angles, while at the poles the density is small. Therefore regardless of the fast rotation, these models give systematically smaller $\dot{L}_{\text {in }}$ than those in the models $E$ and $D$. Also, this is why the model $B$ gives smaller $\dot{L}_{\text {in }}$ than that in the model $C$. Model $A$ is the only one in which the torus is closed (the gas rotates fast at the equator at every $\phi$ angle). This leads to a larger $\dot{L}_{\text {in }}$ than that in model $B$, which is again less affected by the density distribution.

In the models $A, B$ and $C$, the accretion rate and $L_{\text {in }}$ are variable. For model $A$, a characteristic wave pattern can be seen in the specific angular momentum distribution in the equatorial plane (see Fig. 3 at $t^{\prime} \sim 0.09$ ). This behavior is reflected in the variable $\dot{M}_{\text {in }}$ and $\dot{L}_{\text {in }}$, due to the variable radial velocity and nearly constant density at the inner boundary. At time $t^{\prime} \sim 0.16$, when the accretion rate stops varying rapidly, the corresponding 'waves' in specific angular momentum map are smoothed out. The outflow of the gas at this time is suppressed, and the gas accretes onto the center through the poles. As a consequence, the mass flux and angular momentum flux through the inner boundary slightly increase, and the curves are smoother. The subsequent drop of both of these quantities at time $t^{\prime}=0.25$ is caused by the density decrease at the inner edge, when the torus is tilted with respect to the equatorial plane. The density at inner radius increases again at $t^{\prime}=0.29$ and the torus starts precessing.

The behavior of the flow in models $B$ and $C$ is more chaotic. The accretion rate and $\dot{L}_{\text {in }}$ vary in time until the end of our simulations. Also, no precession was detected, because in principle it would be hard to determine the boundaries of the structure which might be precessing. We cannot use a density threshold to define a torus, because such a torus is not a closed ring in these two models.

\section{Discussion}

This paper presented the fourth phase of our study of slightly rotating accretion flows onto black holes (see PB03, Proga \& Begelman 2003b, and Proga 2005 for the first, second and third phase). Here we followed PB03, but we considered 3-D not 2-D axisymmetric effects. As in PB03 we made a few simplifications. For example, we neglected the gravitational field due to the host galaxy, radiative heating and cooling, viscosity and MHD effects. Perhaps the most important simplification we made is neglecting the transport of energy and angular momentum outward as needed to accrete matter with a specific angular momentum higher than $l_{\text {crit }}$. As shown Proga \& Begelman (2003b) for the axisymmetric case, magnetic fields, that can drive the transport, can dramatically alter the flow solution. Still our HD results provide a useful exploratory study of accretion onto black holes as they have revealed unexpected properties and complexity of accretion flows even with simplified physics. To our best knowledge there have not been any MHD simulations of rotating flows where a closed torus was either assumed or failed to form. Our results show that such flows are plausible (see also Loeb 2004) and motivate new simulations. In what follows we summarize and discuss our results.

We have performed numerical 3-D hydrodynamical simulations of slightly rotating, inviscid accretion flows onto a black hole. As in PB03, we attempt to mimic the boundary conditions of classic Bondi accretion flows with the only modifications being the introduction of a small, latitudedependent and also azimuth-dependent angular momentum at the outer boundary and a pseudoNewtonian gravitational potential. The adopted form of the distribution of $l_{\text {spec }}$ the density distribution at infinity to approach spherical symmetry, because the centrifugal force is negligible.

For the latitude-dependent angular momentum, 3-D simulations confirm axisymmetric results. Namely, the material that has too much angular momentum to be accreted forms a thick torus near the equator. Therefore the geometry of the polar funnel, where material is accreted, and the mass accretion rate through it are constrained by the size and shape of the torus but by the outer conditions. However, in 3-D the torus precesses and is non-axisymmetric even for axisymmetric conditions at large radii. For the latitude- and azimuth-dependent angular momentum in the initial conditions, the non-rotating gas near the equator can significantly affect the evolution of the rotating gas. It can prevent closing, in the azimuthal direction, of the rotating gas and the proper torus does not form. In such cases, the mass accretion rate is only slightly less than Bondi rates. 
Simulations with none or a small amount of a non-rotating gas near the equator show that a torus forms and limits the accretion rate. However, a non-rotating gas near the equator can inhibit torus formation and the accretion rate will be close to the Bondi rate. Thus, our simulations show that in 3-D it may be even more difficult than in 2-D to explain the inactive mode of accretion. However, if the torus forms then our simulations show that the torus will precess. This precession may have important consequences in terms of reducing the mass accretion rate. Namely, a precessing torus may produce a precessing jet/wind that will then affect a larger volume of the surrounding material than a non-precessing jet/wind.

Our simulations are in principle relevant to any type of a black hole, which accretes gas with some small angular momentum, because the results should scale with the black hole mass. Note, that the parameters in our models were chosen so that the ratio of the Bondi radius to the Schwarzschild radius was equal to $10^{3}$ and the computational domain was between $1.5 R_{\mathrm{S}}$ and $1.2 \mathrm{R}_{\mathrm{B}}$. In this context, one of the most interesting cases is that of the low luminosity active galaxies, and in particular the Sgr $A^{*}$, for which the supermassive black hole of $M \sim 3.5 \times 10^{6} M_{\odot}$ was identified in the center (Ghez et al. 2005).

The Bondi accretion rate in the Galaxy center inferred from the studies of stellar winds was estimated to be between $10^{-6}$ and $10^{-4} M_{\odot} \mathrm{yr}^{-1}$, while the polarization studies suggest that the actual accretion rate is between $10^{-7}$ and $10^{-5}$ $M_{\odot} \mathrm{yr}^{-1}$ from ROSAT observations (Quataert, Narayan \& Reid 1999; Baganoff et al. 2003; Bower et al. 2005). Although the values overlap, most of the authors agree that in $\operatorname{Sgr} \mathrm{A}^{*}$ the accretion rate onto the black hole is well below the Bondi value. Also, studies of other quiescent AGN suggest that some modification of the Bondi accretion is needed (e.g. Di Matteo et al. 2000).

The hydrodynamical studies of black hole accretion in Sgr $\mathrm{A}^{*}$ which took into account a random distribution of stars were presented in Coker \& Melia (1997). The analytical estimates of the effective angular momentum of the accreting gas performed by Mościbrodzka, Das \& Czerny (2006) were based on the strengths of the stellar winds. Recently, Cuadra, Nayakshin \& Martins (2006) modeled the wind accretion allowing the stars to move on the elliptical orbits. We show here that the accretion rate of less than $40 \%$ of the Bondi value is possible also in models, where the rotationally supported torus does not close, and an asymmetric structure of material being almost spherical on one side of the black hole may persist as a kind of steady-state (model with $\Delta \phi_{0}=120^{\circ}$ ). Only for very small input of the angular momentum gas, corresponding to the total $\Delta \phi_{0} \leq 60^{\circ}$, the accretion flow is still remains almost spherical and the accretion rate does not drop much below the Bondi value.

Perhaps our most intriguing results we found, is the instability and precession of the torus. The precession occurs for the closed rotationally supported torus, which forms for large angular momentum input $330^{\circ} \leq \Delta \phi_{0} \leq 360^{\circ}$. In other words, we found that even a very small asymmetry in the angular momentum distribution (not necessarily in the initial conditions) will lead to the torus misplacement from the equatorial plane and its precession. This happens after a few tens of orbital cycles. For the models where the torus did not form due to a very large content of non rotating gas accreting in purely radial direction, the asymmetric condition makes the quasi-steady configuration very unstable, and rapid fluctuations of the global flow pattern occur on the timescales of a few dynamical cycles. The clumps of gas with large density become misplaced from the equator. For example, in the model with $\Delta \phi_{0}=240^{\circ}$ the misplacement reaches a few tens of degrees and changes in the flow configuration are extremely violent.

The problem of the stability of accretion tori with respect to the axial perturbations was studied in a number of papers. The classical Rayleigh condition for the torus stability (sufficient only for axisymmetric modes) is that the specific angular momentum should not decrease outwards (see e.g. Chandrasekhar 1961). The Kelvin-Helmholtz instability occurs when two superimposed layers of fluid are in a relative motion. When the velocity shear exceeds a critical value, the resulting pressure gradient (from Bernoulli's law) between the peaks and troughs of an interfacial wave overcomes the surface tension and gravity, and the mode grows exponentially.

Papaloizou \& Pringle (1984) studied the stability of the non-axisymmetric modes of the differen- 
tially rotating tori. In this first paper, they limited their considerations to the homentropic tori with constant specific angular momentum, and they found, that all such tori are unstable to the low order modes and the instability occurs on a dynamical timescale. These instabilities are found to be global, i.e. their presence cannot be detected via the local analysis nor from the considerations of axisymmetric modes. In their second paper (Papaloizou \& Pringle 1985), they considered the tori with non-constant specific angular momentum and they found that for low azimuthal number $m$ the instability is driven by a Kelvin-Helmholtz mechanism. The modes are stable in disks with angular velocity decreasing with radius as $\Omega \propto r^{-q}$, if $q>\sqrt{3}$. For high $m$, the modes regain their sonic character, and there exist sonic modes which are driven by both mechanisms. However, their analytical calculations were done in the limit of a Keplerian disk $(q=3 / 2)$. In our models, $q$ is about 1.8. In the innermost parts, the flow is supersonic and compressible. Future work is needed to perform stability analysis for such flow properties.

From the point of view of numerical, multidimensional simulations, as it has been recently discussed by Foglizzo, Galletti \& Ruffert (2005) that it is not always obvious to determine whether the hydrodynamical instabilities are a physical or numerical effect. As shown by our simulations, and confirmed by the tests with smaller Mach numbers (models $M_{100}$ and $M_{300}$ ), the instabilities that lead to the torus precession do not develop in a subsonic flow (see also Mościbrodzka \& Proga 2008). The acoustic instability develops in the 3 -D accretion when the Mach number is large, and for $\gamma=5 / 3$, and it is found to be a physical instability in a strongly supersonic flow. We plan to verify this result for other values of $\gamma$, and perform more detailed resolution tests in the future work.

From the observational point of view, the precessing torus might be relevant to the interpretation of jet emitting sources, provided that the jet axis is always perpendicular to the disk surfaces. Recent observations of radio jets show jet reorientation. One of possible explanation for this reorientation may be the jet precession, as was suggested e.g., for the shape of the source 3C294 (Erlund et al. 2006). Also, the morphology of the BAL quasar $1045+352$ indicates either a pre- cessing jet or an ongoing merger process (KunertBajraszewska \& Marecki 2007). In addition, the morphology of some of the VLBA observed curved jets suggests jet precession because of relatively short timescales (Lister 2006).

We thank Monika Mościbrodzka and Aneta Siemiginowska for helpful discussions and comments. We thank the developers of ZEUS-MP for providing the code publicly available and for support. This work was supported by NASA under grant NNG05GB68G. This work was also supported by the National Science Foundation through TeraGrid resources provided by National Center for Supercomputing Applications under grant AST070019N.

\section{REFERENCES}

Baganoff F.K., et al., 2003, ApJ, 591, 891

Begelman, M.C., \& Meier, D.L. 1982, ApJ, 253, 873

Blandford R., Begelman M., 1999, MNRAS, 303, L1

Bondi H., 1952, MNRAS, 112, 195

Bower G.C., Falcke H., Wright M.C., Backer D.C., 2005, ApJ, 618, L29

Chandrasekhar, S., Hydrodynamic and hydromagnetic stability, 1961, Oxford University Press

Catlett, C. et al. "TeraGrid: Analysis of Organization, System Architecture, and Middleware Enabling New Types of Applications," HPC and Grids in Action, Ed. Lucio Grandinetti, IOS Press 'Advances in Parallel Computing' series, Amsterdam, 2007

Coker R.F., Melia F., 1997, ApJ, 488, L149

Cuadra, J., Nayakshin, S., Springel, V., \& di Matteo, T. 2006, MNRAS, 366, 358

Di Matteo, T., Quataert E., Allen S.W., Narayan R., Fabian, A.C., 2000, MNRAS, 311, 507

Erlund, M.C., Fabian,A.C., Blundell, K.M., Celotti, A., \& Crawford, C.S., 2006, MNRAS, 371,29 
Foglizzo T., Galletti P \& Ruffert M., 2005, A\&A, 435,397

Fragile P.C., Blaes O.M., Annios P., Salmonson J.D., 2007 (astro-ph/0706.4303)

Genzel R., Schödel R., Ott T., et al., 2003, ApJ, 594,812

Ghez A.M., Salim S., Hornstein S.D., et al., 2005, ApJ, 620, 744

Hawley, J.F., \& Balbus, S.A. 2002, ApJ, 573, 749

Hayes J.C., Norman M.L., 2003, ApJS, 147, 197

Ichimaru, S. 1977, ApJ, 214, 840

Igumenshchev, I.V., \& Abramowicz, M.A. 1999, MNRAS, 303, 309

King A.R., Lubow S.H., Ogilvie G.I., Pringle J.E., 2005, MNRAS, 363, 49

Kormendy, J., \& Gebhardt, K. 2001, in AIP Conf. Proc. 586, 20th Texas Symposium on Relativistic Astrophysics, ed. J. C. Wheeler \& H. Martel (Melville: AIP)

Krumholz, McKee, \& Klein, 2005, ApJ, 618, 757

Kunert-Bajraszewska M., Marecki A., 2007, A\&A, 469,437

Lemaster, M.N.., Stone, J.M., Gardiner, T.A., 2007, ApJ, 662, 582

Lister M.L., 2006, AAS 209.0808, Bulletin of the American Astronomical Society, Vol. 38, p.905

Loeb A., 2004, MNRAS, 350, 725

Machida, M., Matsumoto, R., \& Mineshige, S. 2001, PASJ, 53, L1

MacFadyen, A., \& Woosley, S.E. 1999, ApJ, 524, 262

Mościbrodzka M., Das T.K., Czerny B., 2006, MNRAS, 370, 219

Mościbrodzka M. \& Proga, D. 2008, ApJ, in press (arXiv:0801.1076)

Narayan R., Yi I., 1994, ApJ, 428, L13

Narayan, R., \& Yi, I. 1995, ApJ, 444, 231
Paczyński, B. 1998, ApJ, 494, L45

Paczyński, B., \& Abramowicz M.A. 1982, ApJ, 253,897

Paczyński B., Wiita P.J., 1980, A\&A, 88, 23

Papaloizou J.C.B., Pringle J.E., 1984, MNRAS, 208, 721

Papaloizou J.C.B., Pringle J.E., 1985, MNRAS, 213, 799

Papaloizou J.C.B., Szuszkiewicz E., 1994, MNRAS, 268, 29

Proga D., 2005, ApJ, 629, 397

Proga D., Begelman M., 2003a, ApJ, 582, 69 (PB03)

Proga D., Begelman M., 2003b, ApJ, 592, 767

Proga, D., MacFadyen, A. I., Armitage, P. J. \& Begelman, M. C. 2003, ApJ, 599, L5

Quataert, E., \& Gruzinov A. 2000, ApJ, 545, 842

Quataert E., Narayan R., Reid M.J., 1999, ApJ, 517, L101

Rees, M.J., Begelman, M.C., Blandford, R.D., \& Phinney, E.S. 1982, Nature, 295, 17

Stone, J.M., Pringle, J.E., \& Begelman, M.C. 1999, MNRAS, 310, 1002

Schödel R., Ott, T., Genzel, R., Eckart, A., Mouawad, N., Alexander, T., 2003, ApJ, 596, 1015

Stone J.M., Norman M.L., 1992, ApJS, 80, 753

Volonteri M., Sikora M., Lasota J.-P., 2007, ApJ, (astro-ph/0706.3900)

Woosley, S.E. 1993, ApJ, 405, 273

This 2-column preprint was prepared with the AAS IATEX macros v5.2. 
Table 1: Summary of the models for nonaxisymmetric accretion hydrodynamics in $3-\mathrm{D}$

\begin{tabular}{lcccccc}
\hline \hline Model & $\begin{array}{c}\Delta \phi_{0} \\
\left.{ }^{\circ}\right]\end{array}$ & $\begin{array}{c}\text { Resolution } \\
{\left[N_{\mathrm{r}} \times N_{\theta} \times N_{\phi}\right]}\end{array}$ & $\begin{array}{c}T_{\text {end }} \\
{\left[t_{\text {orb }}\left(R_{\mathrm{B}}\right)\right]}\end{array}$ & $\begin{array}{c}\dot{M} \\
{\left[\dot{M}_{\mathrm{B}}\right]}\end{array}$ & Presence of torus & Precession \\
\hline$A_{32}$ & 330 & $140 \times 96 \times 32$ & 0.36 & 0.25 & yes & yes \\
$A_{60}$ & 330 & $140 \times 96 \times 60$ & 0.20 & 0.25 & yes & yes \\
$B_{60}$ & 240 & $140 \times 96 \times 60$ & 0.30 & 0.28 & not closed & - \\
$C_{32}$ & 120 & $140 \times 96 \times 32$ & 0.36 & 0.37 & not closed & - \\
$C_{60}$ & 120 & $140 \times 96 \times 60$ & 0.16 & 0.35 & not closed & - \\
$D_{32}$ & 60 & $140 \times 96 \times 32$ & 0.18 & 0.90 & no & - \\
$E_{32}$ & 30 & $140 \times 96 \times 32$ & 0.14 & 0.98 & no & - \\
$R_{10}$ & 360 & $140 \times 100 \times 10$ & 0.32 & 0.24 & yes & no \\
$R_{32}$ & 360 & $140 \times 96 \times 32$ & 0.36 & 0.24 & yes & yes \\
$R_{60}$ & 360 & $140 \times 96 \times 60$ & 0.36 & 0.24 & yes & yes \\
$M_{100}$ & 360 & $140 \times 96 \times 32$ & 11 & - & yes & no \\
$M_{300}$ & 360 & $140 \times 96 \times 32$ & 2.19 & - & yes & no \\
\hline
\end{tabular}



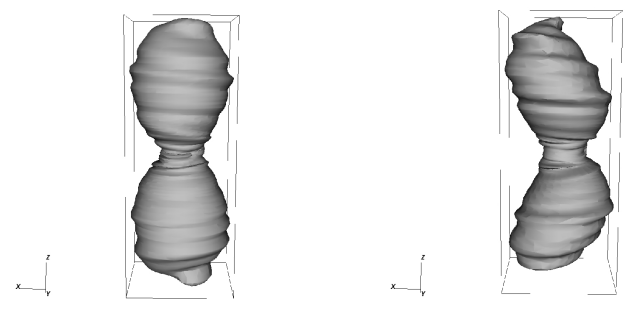

Fig. 1. - The sonic surfaces for the 3-D models with axisymmetric initial conditions (left) and the non-axisymmetric case (right; model $A_{32}$ described in Sec. 3.3 at time $t^{\prime}=0.1$. The extension of the surface in $z$ direction is about 0.065 $R_{\mathrm{B}}$, while in $x$ and $y$ direction the box size is about $0.03 R_{\mathrm{B}}$.
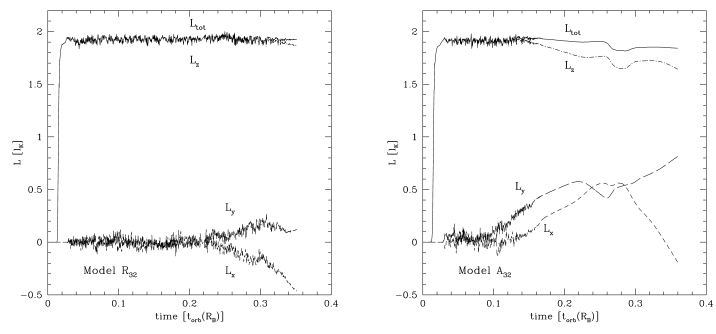

Fig. 2.- The total angular momentum evolution in the initially axisymmetric (left) and nonaxisymmetric (right; model $A_{32}$ described in Sec. 3.3 models. The angular momentum is in the units of Keplerian angular momentum on the inner radius $\left(l_{\mathrm{k}}\right.$, and the plot shows the magnitude of $L_{\text {tot }}$ (solid line) and its components: $L_{\mathrm{x}}$ (short dashed line), $L_{\mathrm{y}}$ (long dashed line) and $L_{\mathrm{x}}$ (dot dashed line).
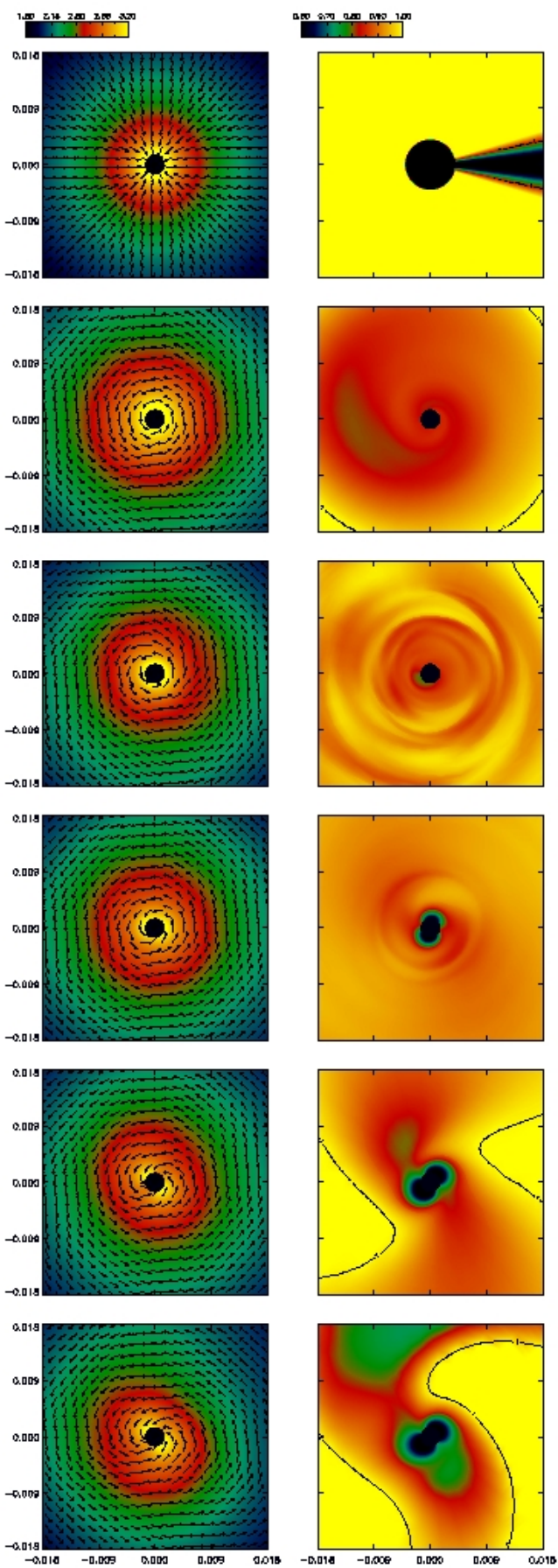

Fig. 3.- The results for model $A_{32}$ (see Tab. 1), for 6 time snapshots, from top to bottom: $t^{\prime}=$ $0.0,0.018,0.09,0.16,0.23$ and 0.29 . The maps show the central region (only upper right map is zoomed-out) in the equatorial plane $\left(\theta=90^{\circ}\right)$ : density and velocity field (left) and specific angular momentum (right). The solid line marks the contour of $l_{\text {spec }}=l_{\text {crit }}$. 

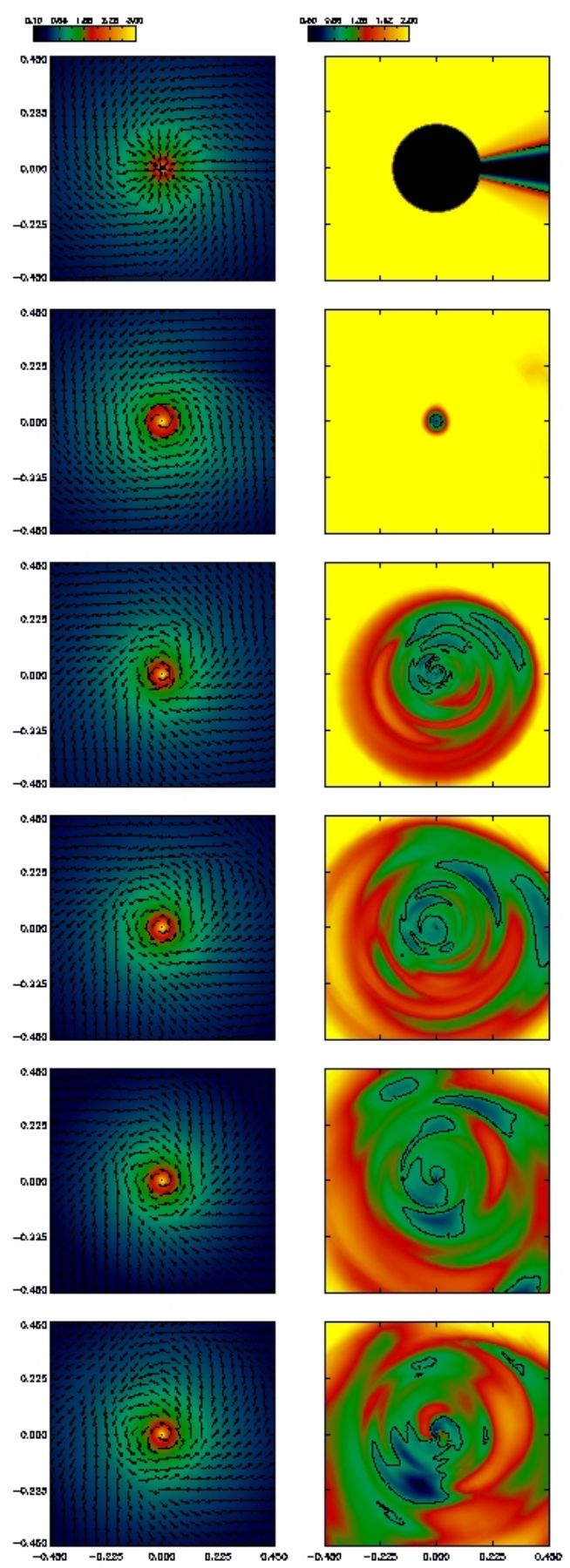

Fig. 4. - The same as in Fig. 3 but the maps show the equatorial plane in a zoom-out (i.e. 20 times larger scale). Note that the color scales are now different than in Fig. 3
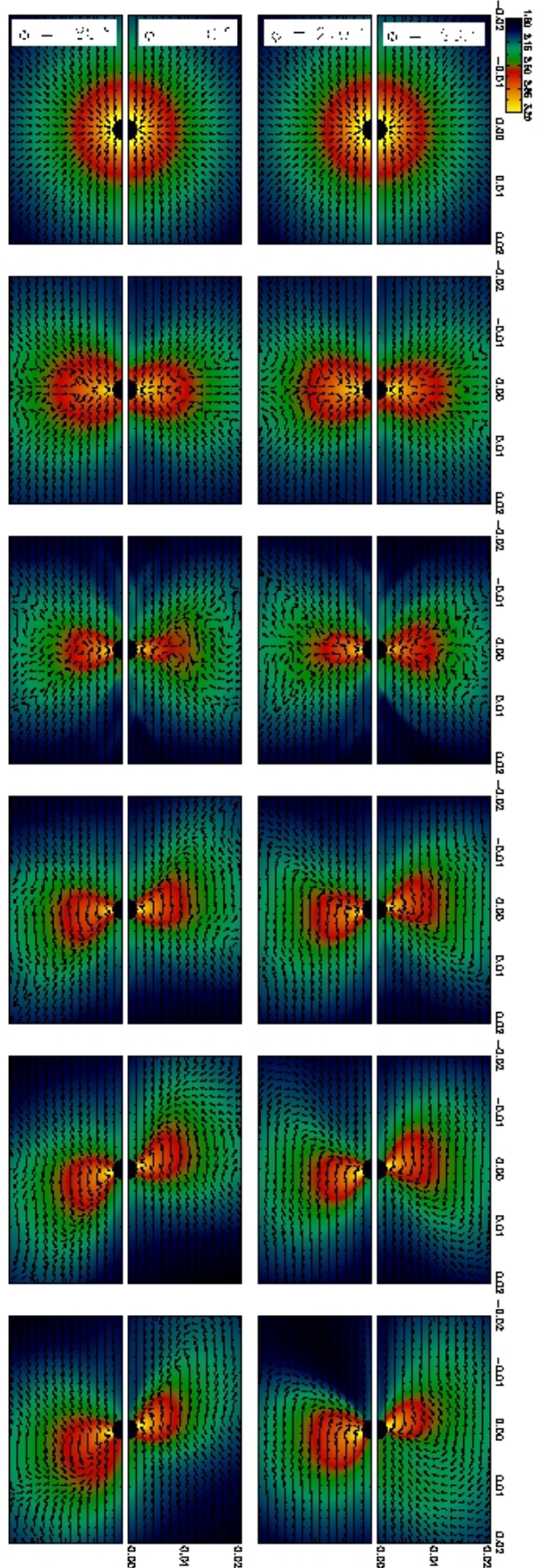

Fig. 5.- The results for model $A_{32}$ (see Tab. 1), plotted in the $r-\theta$ plane, at 4 slices of the $\phi$ angle: $0^{\circ}, 90^{\circ}, 180^{\circ}$ and $270^{\circ}$. The maps show density and velocity fields in the central region. The corresponding times are, from top to bottom: $t^{\prime}=0.0,0.018,0.09,0.16,0.23$ and 0.29 . 

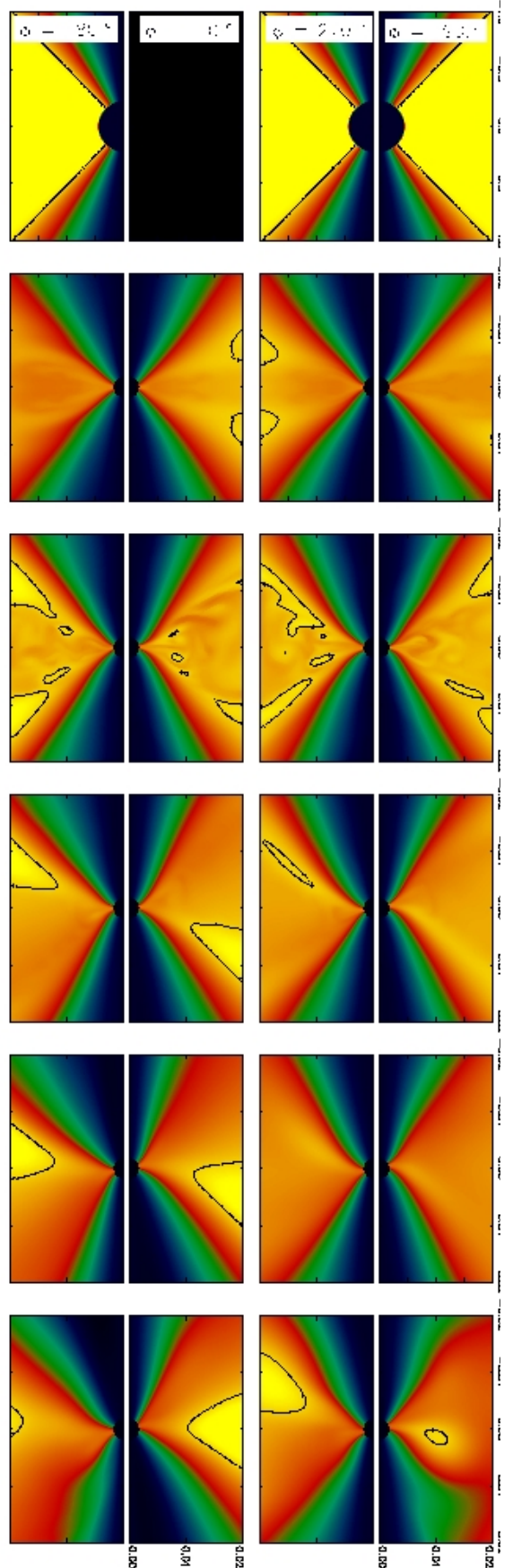

Fig. 6. - The maps of the specific angular momentum for model $A_{32}$ (see Tab. 1), plotted in the $r-\theta$ plane, at 4 slices of the $\phi$ angle: $0^{\circ}$, $90^{\circ}, 180^{\circ}$ and $270^{\circ}$, and at $t^{\prime}=0.0,0.018,0.09$, $0.16,0.23$ and 0.29 (from top to bottom). The maps show the central region (only first map is in zoomed-out). The solid line mark the contour of $l_{\text {spec }}=l_{\text {crit }}$.
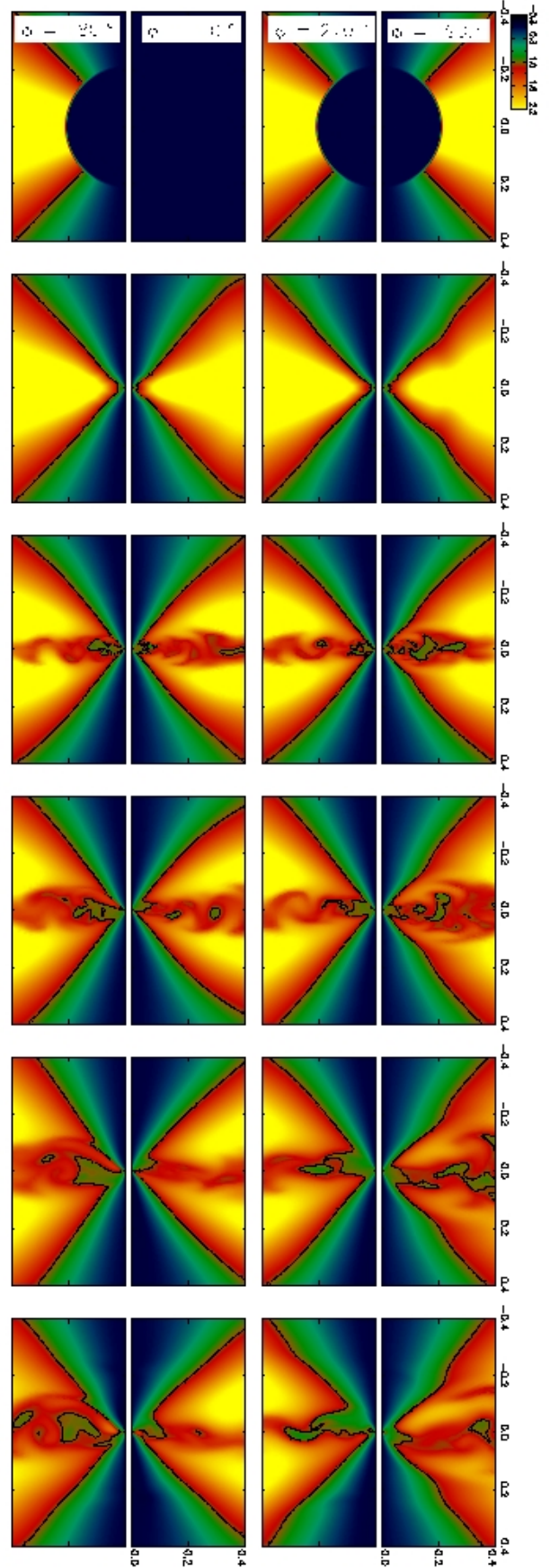

Fig. 7.- The same as in Fig. 6 but all the maps are in zoomed-out. Note that the color scale is now different than in Fig. 6 . 

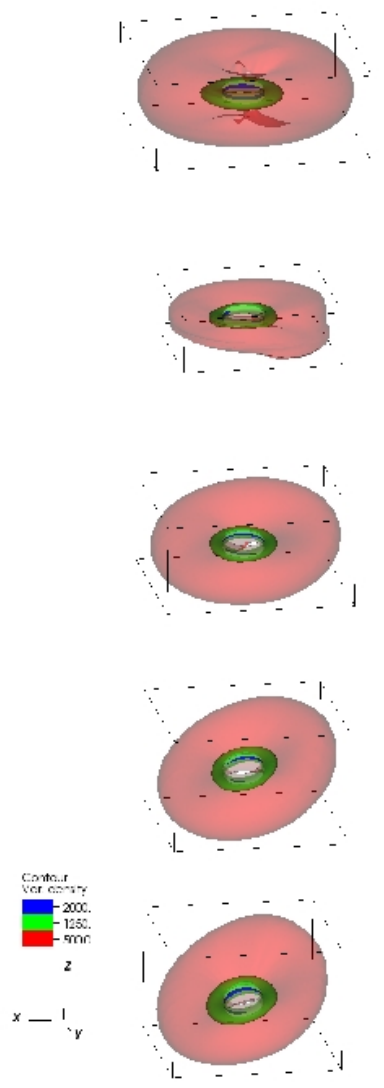

Fig. 8.- Density isosurfaces for five time snapshots: $t^{\prime}=0.018,0.09,0.16,0.23$ and 0.29 , for model $A_{32}$ (see Tab. 11). The colors mark the surfaces of $\rho=500,1250$ and $2000 \rho_{\infty}$. The boxes are scaled to the density isocontour $\rho=500 \rho_{\infty}$ and show the central region of the radius about $0.004 R_{\mathrm{B}}$.

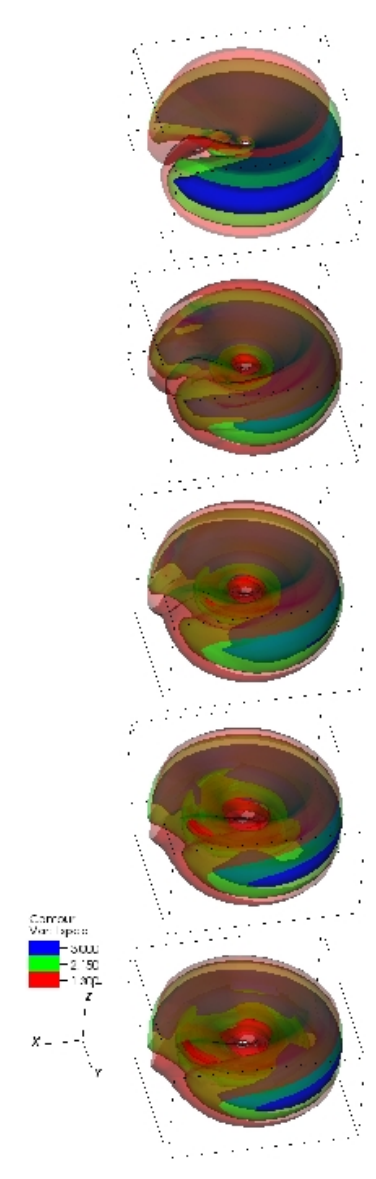

Fig. 9.- Isosurfaces of the specific angular momentum in five time snapshots: $t^{\prime}=0.018,0.09$, $0.16,0.23$ and 0.29 , for model $A_{32}$ (see Tab. 1). The colors mark the surfaces of $l_{\text {spec }} / l_{\text {crit }}=1.3$, 2.15 and 3.0. The boxes are scaled to the isocontour of $l_{\text {spec }} / l_{\text {crit }}=1.3$ and show the region of the radius about $0.6 R_{\mathrm{B}}$. 


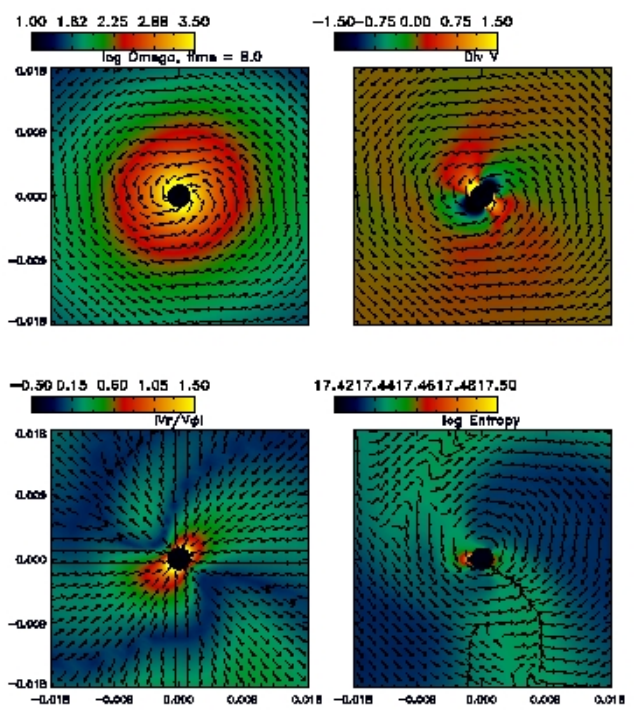

Fig. 10. - The results for model $A_{32}$ (see Tab. 1) plotted in the equatorial plane, at $t^{\prime}=0.29$. The maps show: angular velocity (upper left), velocity divergence (upper right), radial to azimuthal velocity ratio (bottom left) and entropy (bottom right). The arrows denote the directions of velocity vectors with $v_{r}$ and $v_{\phi}$ components (upper two panels), with only $v_{r}$ component (bottom left panel) or vorticity vectors with $w_{r}$ and $w_{\phi}$ components (bottom right panel).

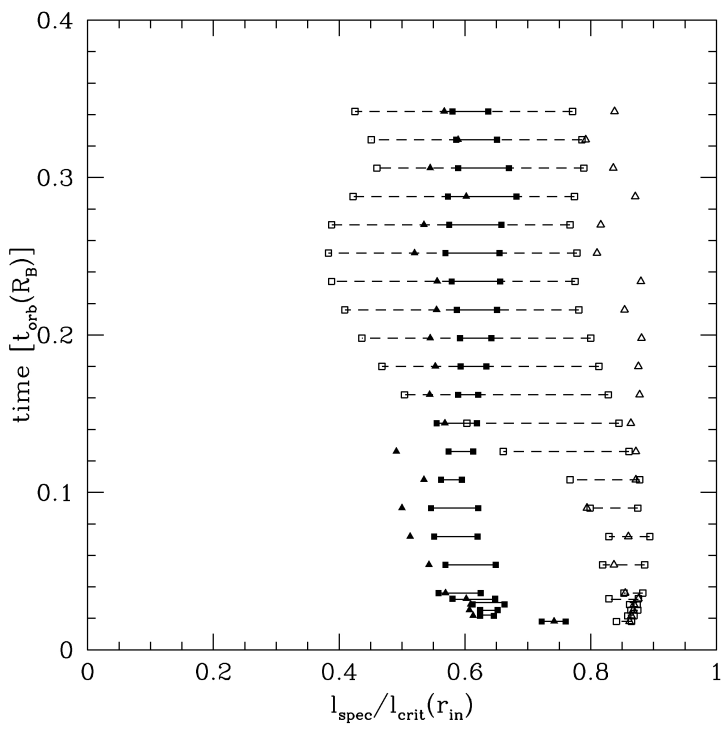

Fig. 11. - The time evolution of the specific angular momentum at the inner radius. The results are shown for the axisymmetric reference model $R$ (triangles) and for the model $A_{32}$ (squares, showing the range of values for different $\phi$-directions). The filled symbols and solid lines denote the $\theta$ averaged results, and the open symbols are for at the equatorial plane. 


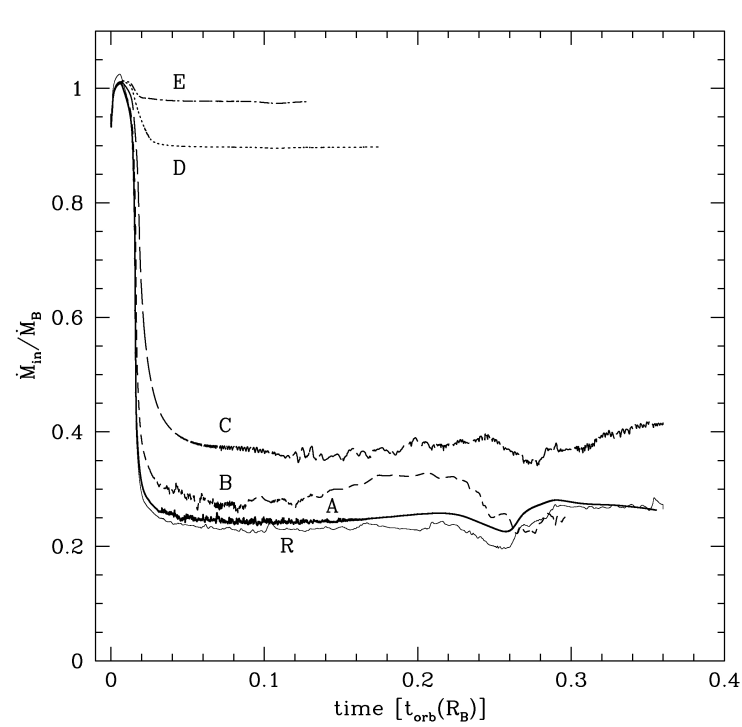

Fig. 12.- Time evolution of the mass accretion rate through the inner boundary $\left(\dot{M}_{\text {in }}\right)$, in units of the Bondi rate $\left(\dot{M}_{\mathrm{B}}\right)$. The non axisymmetric models are: $A$ (thick solid line), $B$ (short dashed line), $C$ (long dashed line), $D$ (dotted line), $E$ (dotdashed line) and reference axisymmetric model $R$ (thin solid line).

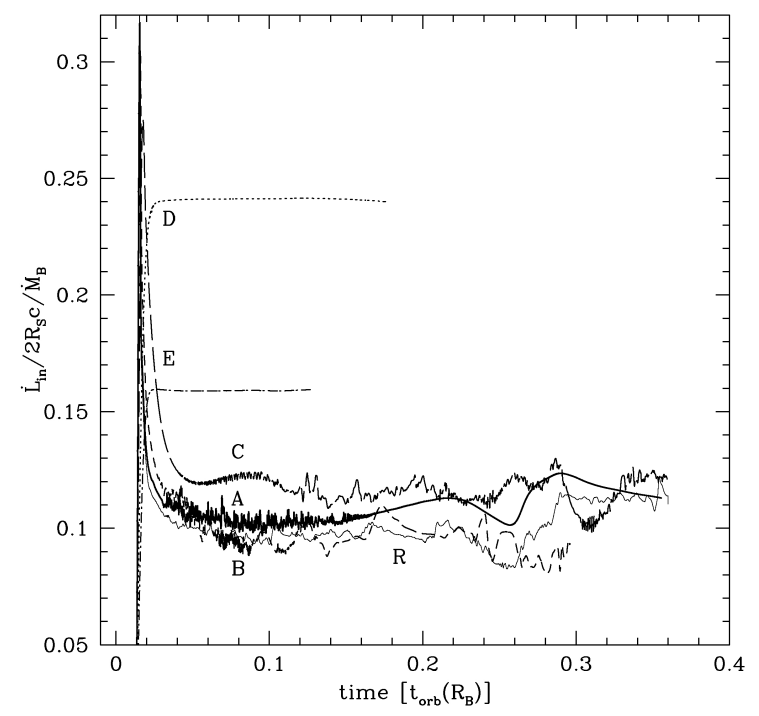

Fig. 13. - Time evolution of the angular momentum flux through the inner boundary, in units of the critical angular momentum times the Bondi accretion rate. The non axisymmetric models are: $A$ (thick solid line), $B$ (short dashed line), $C$ (long dashed line), $D$ (dotted line), $E$ (dot-dashed line) and the reference axisymmetric model $R$ (thin solid line). 


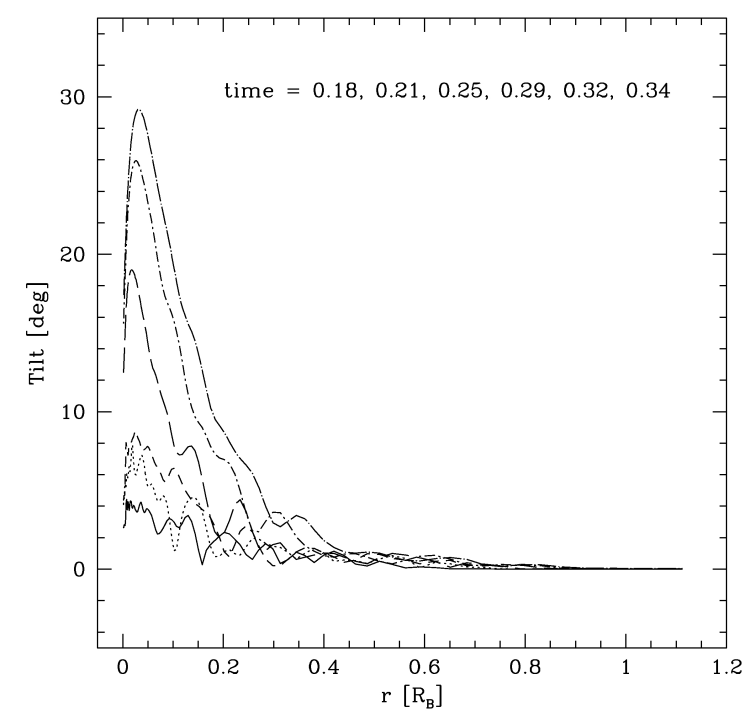

Fig. 14. - The tilt angle $\beta$ (Eq. 7) as a function of radius, for various times in the late phase of the evolution: $t^{\prime}=0.18$ (solid line), 0.21 (dotted line), 0.25 (short dashed line), 0.29 (long dashed line), 0.32 (dotted-short dashed line) and 0.34 (dottedlong dashed line). The initial tilt was zero (initially axisymmetric model).

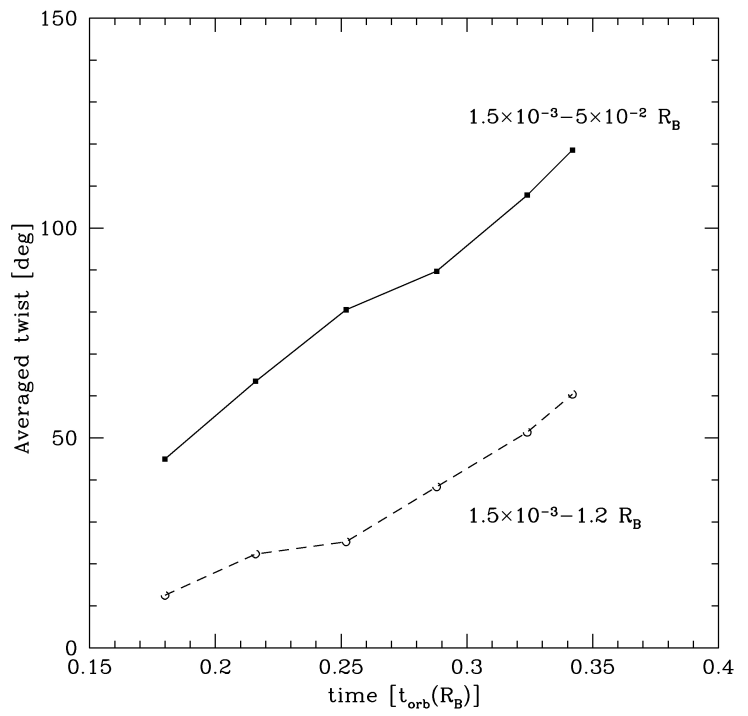

Fig. 15.- The cumulative twist angle $\gamma$ (Eq. 8) as a function of time, for the late phase of the evolution in the initially axisymmetric model. The solid line shows the twist averaged over innermost radii, from $1.5 \times 10^{-3}$ to $5 \times 10^{-2} R_{\mathrm{B}}$, while the dashed line shows the twist averaged over the whole disk, up to $1.2 R_{\mathrm{B}}$. 

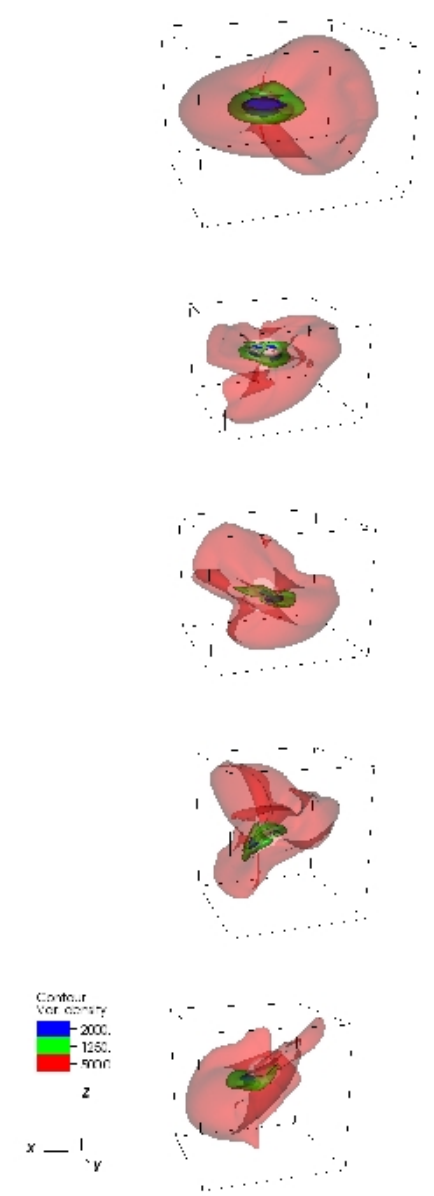

Fig. 16. - Density isosurfaces for five time snapshots: $0.018,0.09,0.16,0.23$ and $0.29 t_{\text {orb }}\left(R_{\mathrm{B}}\right)$, for one of the 'broken torus' models, $B_{60}$ (see Tab. 1). The colors mark the surfaces of $\rho=500,1250$ and $2000 \rho_{\infty}$. The boxes are scaled to the density isocontour $\rho=500 \rho_{\infty}$ and show the central region of the radius about $0.004 R_{\mathrm{B}}$. 